\title{
Long Circuits and Large Euler Subgraphs*
}

\author{
Fedor V. Fomin ${ }^{\dagger} \quad$ Petr A. Golovach ${ }^{\dagger}$
}

\begin{abstract}
An undirected graph is Eulerian if it is connected and all its vertices are of even degree. Similarly, a directed graph is Eulerian, if for each vertex its in-degree is equal to its out-degree. It is well known that Eulerian graphs can be recognized in polynomial time while the problems of finding a maximum Eulerian subgraph or a maximum induced Eulerian subgraph are NP-hard. In this paper, we study the parameterized complexity of the following Euler subgraph problems:

- Large Euler Subgraph: For a given graph $G$ and integer parameter $k$, does $G$ contain an induced Eulerian subgraph with at least $k$ vertices?

- Long Circuit: For a given graph $G$ and integer parameter $k$, does $G$ contain an Eulerian subgraph with at least $k$ edges?

Our main algorithmic result is that LARge Euler SubGraph is fixed parameter tractable (FPT) on undirected graphs. We find this a bit surprising because the problem of finding an induced Eulerian subgraph with exactly $k$ vertices is known to be W[1]-hard. The complexity of the problem changes drastically on directed graphs. On directed graphs we obtained the following complexity dichotomy: LARGE EULER SUBGRAPH is NP-hard for every fixed $k>3$ and is solvable in polynomial time for $k \leq 3$. For Long Circuit, we prove that the problem is FPT on directed and undirected graphs.
\end{abstract}

\section{Introduction}

One of the oldest theorems in Graph Theory is attributed to Euler, and it says that a (undirected) graph admits an Euler circuit, i.e., a closed walk visiting every edge exactly once, if and only if a graph is connected and all its vertices are of even degrees. Respectively, a directed graph has a directed Euler circuit if and only if a graph is (weakly) connected and for each vertex, its in-degree is equal to its out-degree. While checking if a

\footnotetext{
${ }^{*}$ Supported by the European Research Council (ERC) via grant Rigorous Theory of Preprocessing, reference 267959.

${ }^{\dagger}$ Department of Informatics, University of Bergen, PB 7803, 5020 Bergen, Norway, \{fomin, petr.golovach\}@ii.uib.no.
} 
given directed or undirected graph is Eulerian is easily done in polynomial time, the problem of finding $k$ edges (arcs) in a graph to form an Eulerian subgraph is NP-hard. We refer to the book of Fleischner [12] for a thorough study of Eulerian graphs and related topics.

In [5], Cai and Yang initiated the study of parameterized complexity of subgraph problems motivated by Eulerian graphs. Particularly, they considered the following parameterized subgraph and induced subgraph problems:

$k$-CIRCUIT

Parameter: $k$

Input: A (directed) graph $G$ and non-negative integer $k$

Question: Does $G$ contain a circuit with $k$ edges (arcs)?

and

EULER $k$-SUBGRAPH

Parameter: $k$

Input: A (directed) graph $G$ and non-negative integer $k$

Question: Does $G$ contain an induced Euler subgraph with $k$ vertices?

The decision versions of both $k$-CIRCUIT and EUlER $k$-SUBGraPH are known to be the NP-complete [5. Cai and Yang in 5] proved that $k$ CIRCUIT on undirected graphs is FPT. On the other hand, the authors have shown in [14] that EUlER $k$-SUBGRAPH is W[1]-hard. The variant of the problem $(m-k)$-CIRCUIT, where one asks to remove at most $k$ edges to obtain an Eulerian subgraph was shown to be FPT by Cygan et al. [7] on directed and undirected graphs. The problem of removing at most $k$ vertices to obtain an induced Eulerian subgraph, namely EULER $(n-k)$-SUBGRAPH, was shown to be W[1]-hard by Cai and Yang for undirected graphs [5] and by Cygan et al. for directed graphs [7]. Dorn et al. in [8] provided FPT algorithms for the weighted version of Eulerian extension.

In this work we extend the set of results on the parameterized complexity of Eulerian subgraph problems by considering the problems of finding an (induced) Eulerian subgraph with at least $k$ (vertices) edges. We consider the following problems:

LARGe Euler SubGraPH

Parameter: $k$

Input: A (directed) graph $G$ and non-negative integer $k$

Question: Does $G$ contain an induced Euler subgraph with at least $k$ vertices?

and

LONG CIRCUIT

Parameter: $k$

Input: A (directed) graph $G$ and non-negative integer $k$

Question: Does $G$ contain a circuit with at least $k$ edges (arcs)?

The decision version of LONG CIRCUIT was shown to be NP-complete 
by Cygan et al. in [7] and it is not difficult to see that same is true for LARGe Euler Subgraph. Let us note that by plugging-in these observations into the framework of Bodlaender et al. [4, it is easy to conclude that on undirected graphs both problems have no polynomial kernels unless $\mathrm{NP} \subseteq \mathrm{coNP} /$ poly

However, the parameterized complexity of these problems appears to be much more interesting.

Our results. We show that LARGe Euler Subgraph behaves differently for directed and undirected cases. For undirected graphs, we prove that the problem is FPT. We find this result surprising, because very closely related Euler $k$-SubGraph is known to be W[1]-hard [14. The proof is based on a structural result interesting in its own. Roughly speaking, we show that large treewidth certifies containment of a large induced Euler subgraph. For directed graphs, LARGe Euler SUbGrAPH is NP-complete for each $k \geq 4$, and this bound is tight - the problem is polynomial-time solvable for each $k \leq 3$. We also prove that Euler $k$-SUBGRAPH is W[1]-hard for directed graphs. LONG CIRCUIT is proved to be FPT for directed and undirected graphs. Our algorithm is based on the results by Gabow and Nie [15] about the parameterized complexity of finding long cycles. The known and new results about Euler subgraph problems are summarized in Table 1.

\begin{tabular}{|c|c|c|}
\hline & Undirected & Directed \\
\hline$k$-CIRCUIT & FPT [5] & FPT, Prop. 4 \\
\hline EULER $k$-SUBGRAPH & W[1]-hard [14] & W[1]-hard, Thm 3 \\
\hline$(m-k)$-CIRCUIT & FPT 7 & $\begin{array}{ll}\text { FPT } & 7 \\
\end{array}$ \\
\hline EULER $(n-k)$-SUBGRAPH & W[1]-hard [5] & W[1]-hard [7] \\
\hline LONG CIRCUIT & FPT, Thm 7 & FPT, Thm 7 \\
\hline LARGE EULER SubGRAPH & FPT, Thm 2 & $\begin{array}{l}\text { NP-complete for any } k \geq 4 \text {, } \\
\text { Thm } 4 \text {; in } \mathrm{P} \text { for } k \leq 3\end{array}$ \\
\hline
\end{tabular}

Table 1: Parameterized complexity of Euler subgraph problems.

This paper is organised as follows. Section 2 contains basic definitions and preliminaries. In Section 3.1 we show that LARge Euler SubGraph is FPT on undirected graphs. In Section 3.2 we prove that on directed graphs, Euler $k$-Subgraph is W[1]-hard while Large Euler Subgraph is NPcomplete for each $k \geq 4$. In Section 4 we treat LONG CuRCuIT and show that it is FPT on directed and undirected graphs.

\section{Basic definitions and preliminaries}

Graphs. We consider finite directed and undirected graphs without loops or multiple edges. The vertex set of a (directed) graph $G$ is denoted by $V(G)$, the edge set of an undirected graph and the arc set of a directed graph $G$ 
is denoted by $E(G)$. To distinguish edges and arcs, the edge with two endvertices $u, v$ is denoted by $\{u, v\}$, and we write $(u, v)$ for the corresponding arc. For a set of vertices $S \subseteq V(G), G[S]$ denotes the subgraph of $G$ induced by $S$, and by $G-S$ we denote the graph obtained form $G$ by the removal of all the vertices of $S$, i.e., the subgraph of $G$ induced by $V(G) \backslash S$. Let $G$ be an undirected graph. For a vertex $v$, we denote by $N_{G}(v)$ its (open) neighborhood, that is, the set of vertices which are adjacent to $v$. The degree of a vertex $v$ is denoted by $d_{G}(v)=\left|N_{G}(v)\right|$, and $\Delta(G)$ is the maximum degree of $G$. Let now $G$ be a directed graph. For a vertex $v \in V(G)$, we say that $u$ is an in-neighbor of $v$ if $(u, v) \in E(G)$. The set of all in-neighbors of $v$ is denoted by $N_{G}^{-}(v)$. The in-degree $d_{G}^{-}(v)=\left|N_{G}^{-}(v)\right|$. Respectively, $u$ is an out-neighbor of $v$ if $(v, u) \in E(G)$, the set of all out-neighbors of $v$ is denoted by $N_{G}^{+}(v)$, and the out-degree $d_{G}^{+}(v)=\left|N_{G}^{+}(v)\right|$.

For a (directed) graph $G$, a (directed) trail of length $k$ is a sequence $v_{0}, e_{1}, v_{1}, e_{2}, \ldots, e_{k}, v_{k}$ of vertices and edges (arcs resp.) of $G$ such that $v_{0}, \ldots, v_{k} \in V(G), e_{1}, \ldots, e_{k} \in E(G)$, the edges (arcs resp.) $e_{1}, \ldots, e_{k}$ are pairwise distinct, and for $i \in\{1, \ldots, k\}, e_{i}=\left\{v_{i-1}, v_{i}\right\}\left(e_{i}=\left(v_{i-1}, v_{i}\right)\right.$ resp.). A trail is said to be closed if $v_{0}=v_{k}$. A closed (directed) trail is called a (directed) circuit, and it is a (directed) cycle if all its vertices except $v_{0}=v_{k}$ are distinct. Clearly, any cycle is a subgraph of $G$, and it is said that $C$ is an induced cycle of $G$ if $C=G[V(C)]$. A (directed) path is a trail such that all its vertices are distinct. For a (directed) walk (trail, path resp.) $v_{0}, e_{1}, v_{1}, e_{2}, \ldots, e_{k}, v_{k}, v_{0}$ and $v_{k}$ are its end-vertices, and $v_{1}, \ldots, v_{k-1}$ are its internal vertices. For a a (directed) walk (trail, path resp.) with endvertices $u$ and $v$, we say that it is an $(u, v)$-walk (trail, path resp.). We omit the word "directed" if it does not create a confusion. Also we write a trail as a sequence of its vertices $v_{0}, \ldots, v_{k}$.

A connected (directed) graph $G$ is an Euler (or Eulerian) graph if it has a (directed) circuit that contains all edges (arcs resp.) of $G$. By the famous result by Euler (see, e.g., [12]), a connected graph $G$ is an Euler graph if and only if all its vertices have even degrees. Respectively, a connected directed graph $G$ is an Euler directed graph if and only if for each vertex $v \in V(G)$, $d_{G}^{-}(v)=d_{G}^{+}(v)$.

Ramsey numbers. The Ramsey number $R(r, s)$ is the minimal integer $n$ such that any graph on $n$ vertices has either a clique of size $r$ or an independent set of size $s$. By the famous paper by Erdös and Szekeres [10], $R(r, s) \leq\left(\begin{array}{c}r+s-2 \\ r-1\end{array}\right)$.

Parameterized Complexity. Parameterized complexity is a two dimensional framework for studying the computational complexity of a problem. One dimension is the input size $n$ and another one is a parameter $k$. It is said that a problem is fixed parameter tractable (or FPT), if it can be solved in time $f(k) \cdot n^{O(1)}$ for some function $f$, and it is said that a problem is in $\mathrm{XP}$, if it can be solved in time $O\left(n^{f(k)}\right)$ for some function $f$. One of the basic 
assumptions of the Parameterized Complexity theory is the conjecture that the complexity class $\mathrm{W}[1] \neq \mathrm{FPT}$, and it is unlikely that a $\mathrm{W}[1]$-hard problem could be solved in FPT-time. A problem is Para-NP-hard(complete) if it is NP-hard (complete) for some fixed value of the parameter $k$. Clearly, a Para-NP-hard problem is not in XP unless $\mathrm{P}=\mathrm{NP}$. We refer to the books of Downey and Fellows [9, Flum and Grohe [13], and Niedermeier [19] for detailed introductions to parameterized complexity.

Treewidth. A tree decomposition of a graph $G$ is a pair $(X, T)$ where $T$ is a tree and $X=\left\{X_{i} \mid i \in V(T)\right\}$ is a collection of subsets (called bags) of $V(G)$ such that:

1. $\bigcup_{i \in V(T)} X_{i}=V(G)$,

2. for each edge $\{x, y\} \in E(G), x, y \in X_{i}$ for some $i \in V(T)$, and

3. for each $x \in V(G)$ the set $\left\{i \mid x \in X_{i}\right\}$ induces a connected subtree of $T$.

The width of a tree decomposition $\left(\left\{X_{i} \mid i \in V(T)\right\}, T\right)$ is $\max _{i \in V(T)}\left\{\left|X_{i}\right|-\right.$ $1\}$. The treewidth of a graph $G$ (denoted as $\operatorname{tw}(G)$ ) is the minimum width over all tree decompositions of $G$.

Recall that our aim is to prove that LONG CIRCUIT is FPT for directed and undirected graphs and LARGE EulER SubGRAPH is FPT for undirected graphs. Hence, we should argue that these problems are NP-hard. Notice that for exact variants of these problems, i.e., $k$-Circuit and Euler $k$ SUBGRAPH for undirected graphs, the NP-hardness was proved by Cai and Yang [5], and for Long Circuit (for directed and undirected cases), it was shown by Cygan et al. in [7].

Proposition 1. LARge Euler SubgraPh is NP-complete for undirected graphs when $k$ is a part of the input.

Proof. Let $G$ be a $n$-vertex cubic graph. Denote by $G^{\prime}$ the graph obtained by subdividing each edge of $G$. It is straightforward to see that $G^{\prime}$ has an induced Euler subgraph with at least $2 n$ vertices if and only if $G$ is Hamiltonian. As the HAMiltonian CYCLE is known to be NP-complete for cubic planar graphs [16], the result follows.

Finally, we observe that while we obtain FPT results, it is unlikely that these problems have polynomial kernels. Let $G$ be a (directed) graph with $t$ connected components $G_{1}, \ldots, G_{t}$, and let $k$ be a positive integer. Notice that $G$ has a circuit with at least $k$ edges (arcs resp.) if and only if $G_{i}$ has a circuit with at least $k$ edges (arcs resp.) for some $i \in\{1, \ldots, t\}$. Also $G$ has an induced Euler subgraph with at least $k$ vertices if and only if $G_{i}$ has an induced Euler subgraph with at least $k$ vertices for some $i \in$ 
$\{1, \ldots, t\}$. By the results of Bodlaender et al. 4], this observation together with Proposition 1 and the NP-hardness of LONG CIRCUIT [7] immediately imply the following proposition.

Proposition 2. LONG CIRCUIT for directed and undirected graphs and LARGE EUler SUbGRAPH for undirected graphs have no polynomial kernels unless $\mathrm{NP} \subseteq$ coNP / poly.

\section{Large Euler subgraphs}

\subsection{Large Euler subgraphs for undirected graphs}

In this section we show that LARGe Euler SubGraPh is FPT for undirected graphs. Using Ramsey arguments, we prove that if a graph $G$ has sufficiently large treewidth, then $G$ has an induced Euler subgraph on at least $k$ vertices. Then if the input graph has large treewidth, we have a YES-answer. Otherwise, we use the fact that LARGE EulER SUBGraPH can be solved in FPT time for graphs of bounded treewidth. All considered here graphs are undirected.

For a given positive integer $k$, we define the function $f(\ell)$ for integers $\ell \geq 2$ recursively as follows:

- $f(2)=R(k, k-1)+1$,

- $f(\ell)=(k-1)\left(2(\ell-1)\left(f\left(\left\lfloor\frac{\ell}{2}\right\rfloor+1\right)-1\right)+1\right)+1$ for $\ell>2$.

We need the following two lemmas.

Lemma 1. Let $G$ be a graph, and suppose that $s, t$ are distinct vertices joined by at least $f(\ell)$ internally vertex-disjoint paths of length at most $\ell$ in $G$ for some $\ell \geq 2$. Then $G$ has an induced Euler subgraph on at least $k$ vertices.

Proof. Consider the minimum value of $\ell$ such that $G$ has $f(\ell)$ internally vertex disjoint $(s, t)$-paths. We have at least $r=f(\ell)-1$ such paths $P_{1}, \ldots, P_{r}$ that are distinct from the path $s, t$. We assume that each path $P_{i}$ has no chords that either join two internal vertices or an internal vertex and one of the end-vertices, i.e., each internal vertex is adjacent in $G\left[V\left(P_{i}\right)\right]$ only to its two neighbors in $P_{i}$. Otherwise, we can replace $P_{i}$ by a shorter path with all vertices in $V\left(P_{i}\right)$ distinct from the path $s, t$. We consider two cases.

Case 1. $\ell=2$. The paths $P_{1}, \ldots, P_{r}$ are of length two and therefore have exactly one internal vertex. Assume that $u_{1}, \ldots, u_{r}$ are internal vertices of these paths. Because $r=f(2)-1=R(k, k-1)$, the graph $G\left[\left\{u_{1}, \ldots, u_{r}\right\}\right]$ either has a clique $K$ of size $k$ or an independent set $I$ of size at least $k-1$. Suppose that $G$ has a clique $K$. If $k$ is odd, then $G[K]$ is an induced Euler subgraph on $k$ vertices. If $k$ is even, then $G[K \cup\{s\}]$ is an induced Euler 
subgraph on $k+1$ vertices. Assume now that that $I \subseteq\left\{u_{1}, \ldots, u_{r-1}\right\}$ is an independent set of size $k-1$. Let $v \in I$. If $\{s, t\} \in E(G)$ and $k$ is even or $\{s, t\} \notin E(G)$ and $k$ is odd, then $G[I \cup\{s, t\}]$ is an induced Euler subgraph on $k+1$ vertices. Else if $\{s, t\} \notin E(G)$ and $k$ is even or $\{s, t\} \in E(G)$ and $k$ is odd, then $G[I \cup\{s, t\} \backslash\{v\}]$ is an induced Euler subgraph on $k$ vertices.

Case 2. $\ell \geq 3$. We say that paths $P_{i}$ and $P_{j}$ are adjacent if they have adjacent internal vertices. Let $p=f(\lfloor\ell / 2\rfloor+1)$. Suppose that there is an internal vertex $v$ of one of the paths $P_{1}, \ldots, P_{r}$ that is adjacent to at least $2 p-1$ internal vertices of some other distinct $2 p-1$ paths. Then there are $p=f(\lfloor\ell / 2\rfloor+1)$ paths $P_{i_{1}}, \ldots, P_{i_{p}}$ that have respective internal vertices $v_{1}, \ldots, v_{p}$ such that i) $v$ is adjacent to $v_{1}, \ldots, v_{p}$ and ii) either each $v_{j}$ is at distance at most $\lfloor\ell / 2\rfloor$ from $s$ in $P_{i_{j}}$ for all $j \in\{1, \ldots, p\}$ or each $v_{j}$ is at distance at most $\lfloor\ell / 2\rfloor$ from $t$ in $P_{i_{j}}$ for all $j \in\{1, \ldots, p\}$. But then either the vertices $s, v$ or $v, t$ are joined by at least $f(\lfloor\ell / 2\rfloor+1)$ internally vertexdisjoint paths of length at most $\lfloor\ell / 2\rfloor+1<\ell$. This contradicts our choice of $\ell$. Hence, for each $i \in\{1, \ldots, r\}$, any internal vertex of $P_{i}$ has adjacent internal vertices in at most $2 p-2$ other paths, and $P_{i}$ is adjacent to at most $2(\ell-1)(p-1)$ other paths. As $r=(k-1)(2(\ell-1)(p-1)+1)$, there are $k-1$ distinct paths $P_{i_{1}}, \ldots, P_{i_{k-1}}$ that are pairwise non-adjacent, i.e., they have no adjacent internal vertices.

Let $H=G\left[V\left(P_{i_{1}}\right) \cup \ldots \cup V\left(P_{i_{k-1}}\right)\right]$ and $H^{\prime}=G\left[V\left(P_{i_{1}}\right) \cup \ldots \cup V\left(P_{i_{k-2}}\right)\right]$. Notice that by our choice of the paths, $H=P_{i_{1}} \cup \ldots \cup P_{i_{k-1}}$ and $H^{\prime}=$ $P_{i_{1}} \cup \ldots \cup P_{i_{k-2}}$ if $\{s, t\} \notin E(G)$, and $P_{i_{1}} \cup \ldots \cup P_{i_{k-1}}\left(P_{i_{1}} \cup \ldots \cup P_{i_{k-2}}\right.$ resp.) can be obtained from $H$ ( $H^{\prime}$ resp.) by the removal of $\{s, t\}$ if $s, t$ are adjacent. If $\{s, t\} \in E(G)$ and $k$ is even or $\{s, t\} \notin E(G)$ and $k$ is odd, then $H$ is an induced Euler subgraph on at least $k+1$ vertices. Else if $\{s, t\} \notin E(G)$ and $k$ is even or $\{s, t\} \in E(G)$ and $k$ is odd, then $H^{\prime}$ is an induced Euler subgraph on at least $k$ vertices.

For $k \geq 4$, let

$$
\Delta_{k}=1+\frac{(f(3 k-8)-1)\left((f(3 k-8)-2)^{3(k-3)}-1\right)}{f(3 k-8)-3} .
$$

Lemma 2. For $k \geq 4$, any 2-connected graph $G$ with $\Delta(G)>\Delta_{k}$ has an induced Euler subgraph on at least $k$ vertices.

Proof. Let $G$ be a 2-connected graph and let $u$ be a vertex of $G$ with $d_{G}(u)=$ $\Delta(G)$. As $G$ is 2 -connected, $G^{\prime}=G-u$ is connected. Let $v$ be an arbitrary vertex of $N_{G}(u)$. Denote by $T$ a tree of shortest paths from $v$ to all other vertices of $N_{G}(u)$ in $G^{\prime}$.

Claim A. If there is a $(v, w)$-path $P$ of length at least $3(k-3)+1$ in $T$ for some $w \in N_{G}(u)$, then $G$ has an induced Euler subgraph on at least $k$ vertices. 
Proof of Claim $A$. Denote by $v_{0}, \ldots, v_{r}$ the vertices of $N_{G}(u)$ in $P$ in the path order, $v_{0}=v$ and $v_{r}=w$. Let $Q_{1}$ be the union of $\left(v_{0}, v_{1}\right),\left(v_{3}, v_{4}\right), \ldots,\left(v_{\lfloor r / 3\rfloor}, v_{\lfloor r / 3\rfloor+1}\right)$-subpaths of $P$, let $Q_{2}$ be the union of $\left(v_{1}, v_{2}\right),\left(v_{4}, v_{5}\right), \ldots,\left(v_{\lfloor r / 3\rfloor+1}, v_{\lfloor r / 3\rfloor+2}\right)$-subpaths of $P$, and let $Q_{3}$ be the union of $\left(v_{2}, v_{3}\right),\left(v_{5}, v_{6}\right), \ldots,\left(v_{\lfloor r / 3\rfloor-1}, v_{\lfloor r / 3\rfloor}\right)$-subpaths of $P$. Notice that some subpaths can be empty depending whether $r$ modulo 3 is 0,1 or 2 . Observe that $Q_{1}, Q_{2}, Q_{3}$ are edge-disjoint induced subgraphs of $G$. Since $Q_{1} \cup Q_{2} \cup Q_{3}=P$, there is $Q_{i}$ for $i \in\{1,2,3\}$ with at least $k-2$ edges. Then $Q_{i}$ has at least $k-1$ vertices. Let $H=G\left[V\left(Q_{i}\right) \cup\{u\}\right]$. By the definition of $Q_{i}, H$ is a union of induced cycles with one common vertex $u$ such that for different cycles $C_{1}, C_{2}$ in the union, $V\left(C_{1}\right) \cap V\left(C_{2}\right)=\{u\}$ and $\{x, y\} \notin E(G)$ whenever $x \in V\left(C_{1}\right) \backslash\{u\}$ and $y \in V\left(C_{2}\right) \backslash\{u\}$. Hence, $H$ is an Euler graph with at least $k$ vertices.

From now we assume that all $(v, w)$-paths in $T$ have length at most $3(k-3)$ for $w \in N_{G}(u)$.

Claim B. If there is a vertex $w \in V(T)$ with $d_{T}(w) \geq f(3(k-3)+1)$, then $G$ has an induced Euler subgraph on at least $k$ vertices.

Proof of Claim B. Recall that $T$ is a tree of shortest paths from $v$. We assume that $T$ is rooted in $v$. Then the roof defines the parent-child relation on $T$. Let $x_{0}$ be a parent of $w$ (if exists) and let $x_{1}, \ldots, x_{r}$ be the children of $w$. If $w$ has no parent, then $w=v$ and $r \geq f(3(k-3)+1)$. Otherwise, $r \geq$ $f(3(k-3)+1)-1$. Let $y_{0}=v$. For each $i \in\{1, \ldots, r\}$, let $y_{i} \in V(T) \cap N_{G}(u)$ be a descendant of $x_{i}$ in $T$. Denote by $P_{i}$ the unique $\left(w, y_{i}\right)$-path in $T$ for $i \in\{0, \ldots, r\}$. As all $(v, w)$-paths in $T$ have length at most $3(k-3)$ for $w \in N_{G}(u)$, the paths $P_{0}, \ldots, P_{r}$ have length at most $3(k-3)$. Notice that these paths have no common vertices except $w$. Observe now that $y_{0}, \ldots, y_{r}$ are adjacent to $u$ in $G$. Therefore, we have at least $f(3(k-3)+1)$ internally vertex-disjoint $(u, w)$-paths in $G$. By Lemma 1, it implies that $G$ has an induced Euler subgraph on at least $k$ vertices.

To complete the proof of the lemma, it remains to observe that if $\Delta(T)<$ $f(3(k-3)+1)$ and all $(v, w)$-paths in $T$ have length at most $3(k-3)$ for $w \in N_{G}(u)$, then

$$
d_{G}(u) \leq|V(T)| \leq 1+\frac{(f(3 k-8)-1)\left((f(3 k-8)-2)^{3(k-3)}-1\right)}{f(3 k-8)-3}=\Delta_{k} .
$$

Kosowski et al. [18, obtained the following bound for treewidth.

Theorem 1 ([18]). Let $G$ be a graph without induced cycles with at least $k \geq 3$ vertices and let $\Delta(G) \geq 1$. Then $\operatorname{tw}(G) \leq k(\Delta(G)-1)+2$. 
This theorem together with Lemma 2 immediately imply the next lemma.

Lemma 3. Let $G$ be a graph and let $k \geq 4$. If $\operatorname{tw}(G)>k\left(\Delta_{k}-1\right)+2$, then $G$ has an induced Euler subgraph on at least $k$ vertices.

Proof. Suppose that $\operatorname{tw}(G)>k\left(\Delta_{k}-1\right)+2$. Then $G$ has a 2-connected component $G^{\prime}$ with $\operatorname{tw}\left(G^{\prime}\right)>k\left(\Delta_{k}-1\right)+2$. If $\Delta\left(G^{\prime}\right)>\Delta_{k}$, then $G^{\prime}$ has an induced Euler subgraph on at least $k$ vertices by Lemma 2. Otherwise, by Theorem 1, $G^{\prime}$ has an induced cycle on at least $k$ vertices, i.e., an induced Euler subgraph.

Now we show that Large Euler SubGraph is FPT for graphs of bounded treewidth.

Lemma 4. For any positive integer $t$, LARge Euler Subgraph can be solved in linear time for graphs of treewidth at most $t$.

Proof. Recall that the syntax of the monadic second-order logic (MSOL) of graphs includes logical connectives $\vee, \wedge, \neg$, variables for vertices, edges, sets of vertices and edges, and quantifiers $\forall, \exists$ that can be applied to these variables. Besides the standard relations $=, \in, \subseteq$, the syntax includes the relation $\operatorname{adj}(u, v)$ for two vertex variables, which expresses whether two vertex $u$ and $v$ are adjacent, and for a vertex variable $v$ and an edge variable $e$, we have the relation $\operatorname{inc}(v, e)$ which expresses that $v$ is incident with $e$. The counting monadic first-order logic (CMSOL) is an extension of MSOL with the additional predicate $\operatorname{card}_{p, q}(X)$ which expresses whether the cardinality of a set $X$ is $p$ modulo $q$.

By the celebrated Courcelle's theorem [6], any problem that can be expressed in CMSOL can be solved in linear time for graphs of bounded treewidth. Moreover, this result holds also for optimization problems.

Observe that to solve LARge Euler Subgraph for a graph $G$, it is sufficient to find a subset of vertices $U$ of maximum size such that $U$ induces an Euler graph. Clearly, $U$ induces an Euler graph if and only if i) $G[U]$ is connected and ii) each vertex of $G[G]$ has even degree. The both properties can be expressed in CMSOL. The standard way to express connectivity is to notice that $G[U]$ is connected if and only if for any $X \subset U, X \neq \emptyset$ and $X \neq U$, there is an edge $\{x, y\} \in E(G)$ such that $x \in X$ and $y \in U \backslash X$. Then we have to express the property that for any $u \in U, d_{G[U]}(u)=\left|N_{G[U]}(u)\right|$ is even. To do it, it is sufficient to observe that $X=N_{G[U]}(u)$ if and only if $X \subseteq U$ such that i) for any $v \in X,\{u, v\} \in E(G)$, and ii) for any $v \in U$ such that $\{u, v\} \in E(G), v \in X$. Since we can express in CMSOL whether $\left|N_{G[U]}(u)\right|$ is even, the claim follows.

Now we can prove the main result of this section. 
Theorem 2. For any positive integer $k$, LARge Euler Subgraph can be solved in linear time for undirected graphs.

Proof. Clearly, we can assume that $k \geq 3$, as any Euler graph has at least three vertices. If $k=3$, then we can find any shortest cycle in the input graph $G$. It is straightforward to see that if $G$ has no cycles, then we have no Euler subgraph, and any induced cycle is an induced Euler subgraph on at least three vertices. Hence, it can be assumed that $k \geq 4$. We check in linear time whether $\operatorname{tw}(G)>k\left(\Delta_{k}-1\right)+2$ using the Bodlaender's algorithm [3]. If it is so, we solve our problem using Lemma 4. Otherwise, by Lemma 3 , we conclude that $G$ has an induced Euler subgraph on at least $k$ vertices and return a YES-answer.

Notice, that the proof of Theorem 2 is not constructive. In the conclusion of this section we sketch the algorithm that produces an induced Euler subgraph on at least $k \geq 4$ vertices if it exists.

First, for each $\ell \geq 2$, we can test existence of two vertices $s, t$ such that the input graph $G$ has at least $f(\ell)$ internally vertex-disjoint $(s, t)$-paths of length at most $\ell$ in FPT time with the parameter $\ell$ using the color coding technique [17. If we find such a structure for $\ell \leq 3 k-8$, we find an induced Euler subgraph with at least $k$ vertices that is either a clique or a union of $(s, t)$-paths as it is explained in the proof of Lemma 1.

Otherwise, we find all 2-connected components. If there is a 2-connected component $G^{\prime}$ with a vertex $u$ with $d_{G^{\prime}}(u)>\Delta_{k}$, then we find an induced Euler subgraph with at least $k$ vertices that is a union of cycles with the common vertex $u$ using the arguments form the proof of Lemma 2 ,

If all 2-connected components have bounded maximum degrees, we use the algorithm of Kosowski et al. [18] that in polynomial time either finds an induced cycle on at least $k$ vertices or constructs a tree decomposition of width at most $k\left(\Delta_{k}-1\right)+2$. In the fist case we have an induced Euler subgraph on at least $k$ vertices. In the second case the treewidth is bounded, and LARGE Euler SubGRAPH is solved by a dynamic programming algorithm instead of applying Lemma 4.

\subsection{Large Euler subgraphs for directed graphs}

In this section we show that Euler $k$-Subgraph and Large Euler SubGRAPH are hard for directed graphs.

First, we consider Euler $k$-Subgraph. It is straightforward to see that this problem is in XP, since we can check for every subset of $k$ vertices, whether it induces an Euler subgraph. We prove that this problem cannot be solved in FPT time unless FPT $=\mathrm{W}[1]$.

Theorem 3. The EUlER $k$-SUBGRAPH is W[1]-hard for directed graphs. 
Proof. We reduce the Multicolored $k$-Clique problem that is known to be W[1]-hard [11]:

Multicolored $k$-Clique

Parameter: $k$

Input: A $k$-partite graph $G=\left(V_{1} \cup \cdots \cup V_{k}, E\right)$, where $V_{1}, \ldots, V_{k}$ are sets of the $k$-partition

Question: Does $G$ has a clique with $k$ vertices?

Let $G=\left(V_{1} \cup \cdots \cup V_{k}, E\right)$ be an instance of Multicolored $k$-Clique. We construct the directed graph $H$ as follows.

- Construct the copies of $V_{1}, \ldots, V_{k}$.

- For $1 \leq i<j \leq k$ and for each $u \in V_{i}$ and $v \in V_{j}$, if $\{u, v\} \notin E(G)$, then construct an $\operatorname{arc}(u, v)$ for the copies of $u$ and $v$ in $H$.

- For each $i \in\{1, \ldots, k\}$, construct two vertices $x_{i}, y_{i}$, then join $x_{i}$ by arcs with all vertices of $V_{i}$ and join every vertex of $V_{i}$ with $y_{i}$ by an arc.

- Construct $\operatorname{arcs}\left(y_{1}, x_{2}\right),\left(y_{2}, x_{3}\right), \ldots,\left(y_{k}, x_{1}\right)$.

We set $k^{\prime}=3 k$.

We claim that $G$ has a clique with $k$ vertices if and only if $H$ has an induced Euler subgraph with at least $k^{\prime}$ vertices.

Let $K=\left\{v_{1}, \ldots, v_{k}\right\}$ be a clique in $G, v_{i} \in V_{i}$ for $i \in\{1, \ldots, k\}$. Observe that $v_{1}, \ldots, v_{k}$ are pairwise non-adjacent in $H$. Hence, the set of vertices $\left\{x_{1}, v_{1}, y_{1}, \ldots, x_{k}, v_{k}, y_{k}\right\}$ induces a cycle in $H$. Hence, we have an induced Euler subgraph with at least $k^{\prime}$ vertices.

Suppose now that $H$ has an induced Euler subgraph $C$ with at least $k^{\prime}$ vertices. Observe that every directed cycle in $G$ contains the arc $\left(y_{k}, x_{1}\right)$, because if we delete this arc, we obtain a directed acyclic graph. Since any Euler directed graph is a union of arc-disjoint directed cycles (see, e.g., [12]), $C$ is an induced directed cycle. Moreover, for each $i \in\{1, \ldots, k\}, C$ contains at most one vertex of $V_{i}$. Indeed, assume that two vertices $u, v$ of $C$ are in the same set $V_{i}$. Then the $(u, v)$-paths and the $(v, u)$-path in $C$ should contain $\left(y_{k}, x_{1}\right)$, but this is impossible. Because $C$ has $3 k$ vertices, we conclude that $C$ contains exactly one vertex $v_{i}$ from each $V_{i}$ for $i \in\{1, \ldots, k\}$, and $x_{i}, y_{i} \in V(C)$ for $i \in\{1, \ldots, k\}$. Then $C=H\left[\left\{x_{1}, v_{1}, y_{1}, \ldots, x_{k}, v_{k}, y_{k}\right\}\right]$. Since $C$ is an induced cycle, $v_{1}, \ldots, v_{k}$ are pairwise non-adjacent in $H$. Then $\left\{v_{1}, \ldots, v_{k}\right\}$ is a clique in $G$.

For LARge Euler Subgraph for directed graphs, we prove that this problem is Para-NP-complete.

Theorem 4. For any $k \geq 4$, Large Euler Subgraph is NP-complete for directed graphs. 
Proof. We reduce to the 3-SATisfiability problem. It is known [2] that this problem is NP-complete even if each variable is used in exactly 4 clauses: 2 times in positive and 2 times in negations.

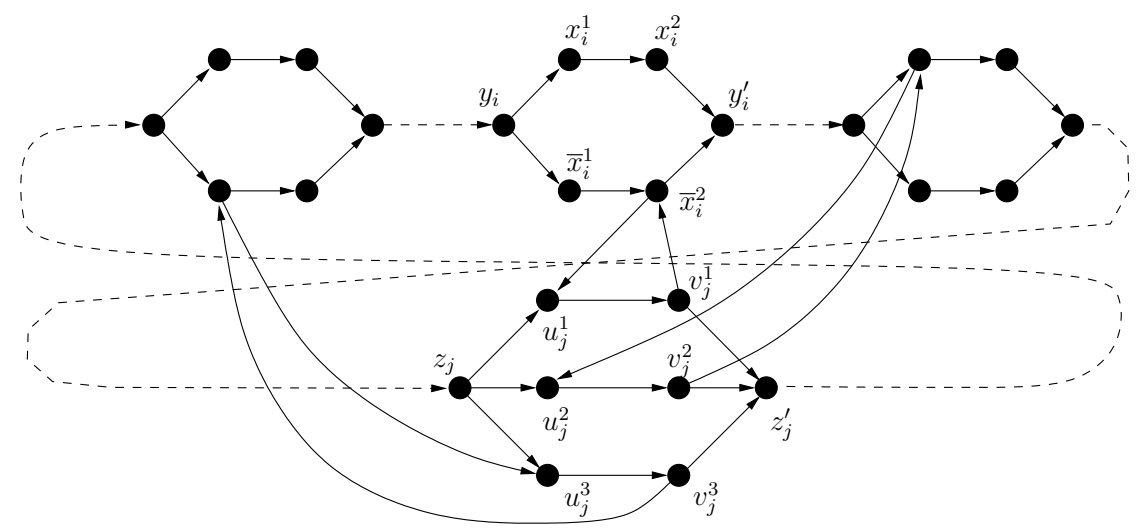

Figure 1: Construction of $G$.

Suppose that Boolean variables $x_{1}, \ldots, x_{n}$ and clauses $C_{1}, \ldots, C_{m}$ compose an instance of 3-SATISFIABILITY such that each variable is used in exactly 4 clauses: 2 times in positive and 2 times in negations. Let $\phi=C_{1} \wedge \ldots \wedge C_{m}$. Without loss of generality we assume that $k \leq 4(n+m)$. We construct the directed graph $G$ as follows (see Fig. 1).

- For each $i \in\{1, \ldots, n\}$, construct vertices $y_{i}, y_{i}^{\prime}, x_{i}^{1}, x_{i}^{2}, \bar{x}_{i}^{1}, \bar{x}_{i}^{2}$ and arcs $\left(y_{i}, x_{i}^{1}\right),\left(x_{i}^{1}, x_{i}^{2}\right),\left(x_{i}^{2}, y_{i}^{\prime}\right),\left(y_{i}, \bar{x}_{i}^{1}\right),\left(\bar{x}_{i}^{1}, \bar{x}_{i}^{2}\right),\left(\bar{x}_{i}^{2}, y_{i}^{\prime}\right)$.

- For each $i \in\{2, \ldots, n\}$, construct $\left(y_{i-1}^{\prime}, y_{i}\right)$.

- For each $j \in\{1, \ldots, m\}$, construct vertices $z_{j}, z_{j}^{\prime}, u_{j}^{1}, u_{j}^{2}, u_{j}^{3}, v_{j}^{1}, v_{j}^{2}, v_{j}^{3}$ and $\operatorname{arcs}\left(z_{j}, u_{j}^{1}\right),\left(u_{j}^{1}, v_{j}^{1}\right),\left(v_{j}^{1}, z_{j}^{\prime}\right),\left(z_{j}, u_{j}^{2}\right),\left(u_{j}^{2}, v_{j}^{2}\right),\left(v_{j}^{2}, z_{j}^{\prime}\right),\left(z_{j}, u_{j}^{3}\right)$, $\left(u_{j}^{3}, v_{j}^{3}\right),\left(v_{j}^{3}, z_{j}^{\prime}\right)$.

- For each $j \in\{2, \ldots, m\}$, construct $\left(z_{j-1}^{\prime}, z_{j}\right)$.

- Construct $\left(y_{n}^{\prime}, z_{1}\right),\left(z_{m}^{\prime}, y_{1}\right)$.

- For each $j \in\{1, \ldots, m\}$, let $C_{j}=w_{1} \vee w_{2} \vee w_{3}$. For $h \in\{1,2,3\}$,

- if $w_{h}=x_{i}$ for some $i \in\{1, \ldots, n\}$ and $w_{h}$ is the fist occurrence of the literal $x_{i}$ in $\phi$, then construct $\left(x_{i}^{1}, u_{j}^{h}\right),\left(v_{j}^{h}, x_{i}^{1}\right)$;

- if $w_{h}=x_{i}$ for some $i \in\{1, \ldots, n\}$ and $w_{h}$ is the second occurrence of the literal $x_{i}$ in $\phi$, then construct $\left(x_{i}^{2}, u_{j}^{h}\right),\left(v_{j}^{h}, x_{i}^{2}\right)$;

- if $w_{h}=\bar{x}_{i}$ for some $i \in\{1, \ldots, n\}$ and $w_{h}$ is the fist occurrence of the literal $\bar{x}_{i}$ in $\phi$, then construct $\left(\bar{x}_{i}^{1}, u_{j}^{h}\right),\left(v_{j}^{h}, \bar{x}_{i}^{1}\right)$; 
- if $w_{h}=\bar{x}_{i}$ for some $i \in\{1, \ldots, n\}$ and $w_{h}$ is the second occurrence of the literal $\bar{x}_{i}$ in $\phi$, then construct $\left(\bar{x}_{i}^{2}, u_{j}^{h}\right),\left(v_{j}^{h}, \bar{x}_{i}^{2}\right)$.

We claim that $\phi$ can be satisfied if and only if $G$ has an induced Euler subgraph with at least $k$ vertices.

For $i \in\{1, \ldots, n\}$, let $P_{i}=\left\{y_{i}, x_{i}^{1}, x_{i}^{2}, y_{i}^{\prime}\right\}$ and $\bar{P}_{i}=\left\{y_{i}, \bar{x}_{i}^{1}, \bar{x}_{i}^{2}, y_{i}^{\prime}\right\}$. For $j \in\{1, \ldots, n\}$ and $h \in\{1,2,3\}, Q_{j}^{h}=\left\{z_{j}, u_{j}^{h}, v_{j}^{h}, z_{j}^{\prime}\right\}$. Let also $Z_{j}=$ $\left\{z_{1}, \ldots, z_{m}\right\}$ and $Z_{j}^{\prime}=\left\{z_{1}^{\prime}, \ldots, z_{m}^{\prime}\right\}$.

Suppose that the variables $x_{1}, \ldots, x_{n}$ have values such that $\phi$ is satisfied. We construct the set of vertices $U$ as follows:

- for $i \in\{1, \ldots, n\}$, if $x_{i}=$ true, then the vertices of the set $P_{i}$ are included in $U$, and $\bar{P}_{i}$ is included otherwise;

- for $j \in\{1, \ldots, m\}$, if $C_{j}=w_{1} \vee w_{2} \vee w_{3}$, then we choose a literal $w_{h}=$ true in $C_{j}$ and include $Q_{j}^{h}$ in $U$.

Observe that $U$ induces a cycle in $G$ and $|U|=4(n+m) \geq k$. Then $G[U]$ is an induced Euler subgraph on at least $k$ vertices.

Assume now that a set $U \subseteq V(G)$ induces an Euler graph $H=G[U]$ and $|U| \geq k$.

Since $k \geq 4, U \cap\left(Z \cup Z^{\prime}\right) \neq \emptyset$ because $G-\left(Z \cup Z^{\prime}\right)$ has no cycles except vertex-disjoint triangles. Suppose that some $z_{j} \in U$. If $j=1$, then $y_{n}^{\prime} \in U$, and if $j>1$, then $z_{j-1}^{\prime} \in U$. Also exactly one of the vertices $u_{j}^{1}, u_{j}^{2}, u_{j}^{3}$ is in $U$. Let $u_{j}^{h} \in U$. Then $v_{j}^{h} \in U$. Further, either $z_{j}^{\prime} \in U$ or some vertex $x_{i}^{p} \in U$ or $\bar{x}_{i}^{p} \in U$. But if $x_{i}^{p} \in U$ or $\bar{x}_{i}^{p} \in U, d_{H}^{-} u_{j}^{h}=2>1=d_{H}^{+}\left(u_{j}^{h}\right)$. Hence, $z_{j}^{\prime} \in U$. If $j=m$, then $y_{1} \in U$, and if $j<m$, then $z_{j+1} \in U$. Suppose now that some $z_{j}^{\prime} \in U$. If $j=m$, then $y_{1} \in U$, and if $j<m$, then $z_{j+1} \in U$. Also exactly one of the vertices $v_{j}^{1}, v_{j}^{2}, v_{j}^{3}$ is in $U$. Let $v_{j}^{h} \in U$. Then $u_{j}^{h} \in U$. Further, either $z_{j} \in U$ or some vertex $x_{i}^{p} \in U$ or $\bar{x}_{i}^{p} \in U$. But if $x_{i}^{p} \in U$ or $\bar{x}_{i}^{p} \in U, d_{H}^{-} v_{j}^{h}=1<2=d_{H}^{+}\left(v_{j}^{h}\right)$. Hence, $z_{j} \in U$. If $j>1$, then $z_{j-1}^{\prime} \in U$. We have that for each $j \in\{1, \ldots, m\}$, exactly one $Q_{h} \subseteq U$ for $h \in\{1,2,3\}$, and for $h^{\prime} \in\{1,2,3\} \backslash\{h\}, u_{j}^{h^{\prime}}, v_{j}^{h^{\prime}} \notin U$. Also we have that $y_{1} \in U$.

Now we consequently consider $i=1, \ldots, n$. We already know that $y_{1} \in$ $U$. Assume inductively that $y_{i} \in U$. Then exactly one of the vertices $x_{i}^{1}, \bar{x}_{i}^{1}$ is in $U$. Assume that $x_{i}^{1}$ is in $U$ as another case is symmetric. Then either $x_{i}^{2}$ or some vertex $u_{j}^{h}$ should be in $U$. But if $u_{j}^{h} \in U$, then because $z_{j} \in U$, $d_{H}^{-}\left(u_{j}^{h}\right)=2>d_{H}^{+}\left(u_{j}^{h}\right)$ and this is impossible for an Euler graph. Then $x_{j}^{2} \in U$. By the same arguments we show that $y_{i}^{\prime} \in U$. If $i<n$, then $y_{i+1} \in U$, and we can proceed with our inductive arguments. We conclude that for each $i \in\{1, \ldots, n\}$, either $P_{i} \subseteq U$ or $\bar{P}_{i} \subseteq U$, and if $P_{i} \subseteq U$ ( $\bar{P}_{i} \subseteq U$ resp. $)$, then $\bar{x}_{i}^{1}, \bar{x}_{i}^{2} \notin U\left(x_{i}^{1}, x_{i}^{2} \notin U\right.$ resp. $)$. Moreover, if $P_{i} \subseteq U$ $\left(\bar{P}_{i} \subseteq U\right.$ resp.) and $Q_{j}^{h} \subseteq U$, then $H$ has no arcs between the vertices of these two sets. 
We define the truth assignment for the variables $x_{1}, \ldots, x_{n}$ as follows: for each $i \in\{1, \ldots, n\}$, if $P_{i} \subseteq U$, then $x_{i}=$ false, and $x_{i}=$ true otherwise. We claim that $\phi$ is satisfied by this assignment. To show it, consider a clause $C_{j}=w_{1} \vee w_{2} \vee w_{3}$. We know that there is $h \in\{1,2,3\}$ such that $Q_{j}^{h} \subseteq U$. Assume that $w_{h}=x_{i}$ (the case $w_{h}=\bar{x}_{i}$ is symmetric). Then $Q_{i}^{h}$ is joined by $\operatorname{arcs}$ in $G$ with $P_{i}$. It follows that $P_{i}$ was not included in $U$, i.e., $\bar{P}_{i} \subseteq U$ and $x_{i}=$ true. Hence, $w_{h}=$ true.

We proved that Large Euler Subgraph is NP-complete for directed graphs for $k \geq 4$. In the conclusion of this section we observe that the bound $k \geq 4$ is tight unless $\mathrm{P}=\mathrm{NP}$.

Proposition 3. LARGe Euler SubGraph can be solved in polynomial time for $k \leq 3$.

Proof. For $k=1$, the problem is trivial. If $k=2$, then any shortest cycle $C$ in a directed graph $G$ is an induced Euler subgraph of $G$ with at least two vertices, and $G$ has no induced Euler subgraph if $G$ is a directed acyclic graph. Hence, it remains to consider $k=3$.

Suppose that $H$ is an induced Euler subgraph of a directed graph $G$, and $H$ has at least three vertices. Denote by $H^{\prime}$ the graph obtained from $H$ by the deletion of all pairs of opposite arcs, i.e., for each pair of vertices $x, y$ such that $(x, y),(y, x) \in E(H)$, we delete $(x, y),(y, x)$. Clearly, for any $v \in V\left(H^{\prime}\right)$, $d_{H^{\prime}}^{-}(v)=d_{H^{\prime}}^{+}(v)$. If $H^{\prime}$ is empty, then because $|V(H)| \geq 3$, there are three distinct vertices $x, y, z \in V(H)$ such that $(x, y),(y, x),(y, z),(z, y) \in E(H)$ and either $(x, z),(z, x) \in E(H)$ or $(x, z),(z, x) \notin E(H)$. Then $G[\{x, y, z\}]$ is an Euler subgraph of $G$. If $H^{\prime}$ is non-empty, then $H^{\prime}$ has a shortest cycle $C$. Because $H^{\prime}$ has no cycles of length two, $G[V(C)]$ is an induced Euler subgraph with at least three vertices.

We conclude that LARge Euler Subgraph can be solved for $k=3$ as follows. If $G$ has three distinct vertices $x, y, z$ such that $(x, y),(y, x),(y, z),(z, y) \in E(G)$ and either $(x, z),(z, x) \in E(G)$ or $(x, z),(z, x) \notin E(G)$, then $G[\{x, y, z\}]$ is an Euler subgraph of $G$. Otherwise, let $G^{\prime}$ be the graph obtained from $G$ by the deletion of all pairs of opposite arcs. We find a shortest cycle $C$ in $G^{\prime}$, and we have that $G[V(C)]$ is an induced Euler subgraph with at least three vertices. Finally, if $G^{\prime}$ is a directed acyclic graph, then we return a NO-answer.

\section{Long circuits}

In this section we show that LONG CIRCUIT problem is FPT for directed and undirected graphs. 
AT LEAST $k$ AND AT MOST $k$-CIRCUIT

Parameter: $k^{\prime}$

Input: A (directed) graph $G$ and non-negative integers $k, k^{\prime}, k \leq k^{\prime}$

Question: Does $G$ contain a circuit with at least $k$ and at most $k^{\prime}$ edges $(\operatorname{arcs})$ ?

Clearly, we can solve this problem in FPT time for undirected graphs applying the algorithm by Cai and Yang [5] for $r$-CIRCUIT for each $r \in$ $\left\{k, \ldots, k^{\prime}\right\}$. For directed graphs, we can use the same approach based on the color coding technique introduced by Alon, Yuster and Zwick [1]. For completeness, we sketch the proof here.

Lemma 5. The AT LEAST $k$ AND AT MOST $k^{\prime}$-CIRCUIT problem can be solved in $2^{O\left(k^{\prime}\right)} \cdot n m$ expected time and in $2^{O\left(k^{\prime}\right)} \cdot n m \log n$ worst-case time for (directed) graphs with $n$ vertices and $m$ edges (arcs).

Proof. As the algorithms for directed and undirected graphs are basically the same, we consider here the directed case. For simplicity, we solve the decision problem, but the algorithm can be easily modified to obtain a circuit of prescribed size if it exists.

Let $G$ be a directed graph with $n$ vertices and $m$ arcs.

First, we describe the randomized algorithm. We color the arcs of $G$ by $k^{\prime}$ colors $1, \ldots, k^{\prime}$ uniformly at random independently from each other. Now we are looking for a colorful circuit in $G$ that has at least $k$ arcs, i.e., for a circuit such that all arcs are colored by distinct colors.

To do it, we apply the dynamic programming across subsets. We choose an initial vertex $u$ and try to construct a circuit that includes $u$. For a set of colors $X \subseteq\left\{1, \ldots, k^{\prime}\right\}$, denote by $U(X)$ the set of vertices $v \in V(G)$ such that there is a $(u, v)$-trail with $|X|$ edges colored by distinct colors from $X$. It is straightforward to see that $U(\emptyset)=\{u\}$. For $X \neq \emptyset, v \in U(X)$ if and only if $v$ has an in-neighbor $w \in N_{G}^{-}(v)$ such that $(w, v)$ is colored by a color $c \in X$ and $w \in U(X \backslash\{c\})$. We consequently construct the sets $U(X)$ for $X$ with $1,2, \ldots, k^{\prime}$ elements. We stop and return a YES-answer if $u \in U(X)$ for some $X$ of size at least $k$. Notice that the sets $U(X)$ can be constructed in time $O\left(k^{\prime} 2^{k^{\prime}} \cdot m\right)$. Since we try all possibilities to select $u$, the running time is $O\left(k^{\prime} 2^{k^{\prime}} \cdot m n\right)$.

Now we observe that for any positive number $p<1$, there is a constant $c_{p}$ such that after running our randomized algorithm $c_{p} 2^{O\left(k^{\prime}\right)}$ times, we either get a YES-answer or can claim that with probability $p G$ has no directed circuit with at least $k$ and at most $k^{\prime}$ arcs.

This algorithm can be derandomized by the technique proposed by Alon, Yuster and Zwick [1. To do it, we replace random colorings by a family of at most $2^{O\left(k^{\prime}\right)} \log n$ hash functions that can be constructed in time $2^{O\left(k^{\prime}\right)}$. $m \log n$.

If we set $k^{\prime}=k$, then Lemma 5 immediately implies the following propo- 
sition. Notice that it was proved in [5] for undirected graphs.

Proposition 4. The $k$-CIRCUIT problem can be solved in $2^{O(k)} \cdot n m$ expected time and in $2^{O(k)} \cdot n m \log n$ worst-case time for (directed) graphs with $n$ vertices and $m$ edges (arcs).

Gabow and Nie in [15] considered the LONG CyCLE problem:

LONG CYCLE

Parameter: $k$

Input: A (directed) graph $G$ and a positive integer $k$

Question: Does $G$ contain a cycle with at least $k$ edges (arcs)?

In particular, they proved the following theorem.

Theorem 5 ([15]). The LONG CYCLE problem can be solved in $2^{O(k \log k)} \cdot n m$ expected time and in $2^{O(k \log k)} \cdot n m \log n$ worst-case time for directed graphs with $n$ vertices and $m$ arcs.

Let us recall that a fundamental cycle in undirected graph is formed from a spanning tree and a nontree edge. For undirected graphs, it is slightly more convenient to use the structural result by Gabow and Nie.

Theorem 6 ([15]). In a connected undirected graph having a cycle with $k$ edges, either every depth-first search tree has a fundamental cycle with at least $k$ edges or some cycle with at least $k$ edges has at most $2 k-4$ edges.

We need the following observation.

Lemma 6. Let $G$ be a (directed) graph without cycles of length at least $k$. If $G$ has a circuit with at least $k$ edges (arcs resp.), then $G$ has a circuit with at least $k$ and at most $2 k-2$ edges (arcs resp.).

Proof. Let $C$ be a circuit in $G$. It is well-known (see, e.g., [12]) that $C$ is a union of edge-disjoint (arc-disjoint) cycles $C_{1}, \ldots, C_{r}$. Moreover, it can be assumed that for any $i \in\{1, \ldots, r\}$, the circuit $C_{1} \cup \ldots \cup C_{i}$ is connected. Suppose now that $C$ is a circuit with at least $k$ edges (arcs resp.) that has minimum length. Then the circuit $C^{\prime}=C_{1} \cup \ldots \cup C_{r-1}$ has at most $k-1$ edges (arcs resp.). Since $G$ has no cycles of length at least $k, C_{r}$ has at most $k-1$ edges (arcs resp.). Thus $C$ has at most $2 k-2$ edges (arcs resp.).

Now we are ready to prove the main result of this section.

Theorem 7. The LONG CIRCUIT problem can be solved in $2^{O(k \log k)} \cdot n m$ expected time and in $2^{O(k \log k)} \cdot n m \log n$ worst-case time for directed graphs with $n$ vertices and $m$ arcs, and in $2^{O(k)} \cdot n m$ expected time and in $2^{O(k)}$. $n m \log n$ worst-case time for undirected graphs with $n$ vertices and $m$ edges. 
Proof. First, we consider directed graphs. Let $G$ be a directed graph. By Theorem 5. we can check whether $G$ has a cycle with at least $k$ arcs. If we find such a cycle $C$, then $C$ is a circuit with at least $k$ arcs, and we have a YES-answer. Otherwise, we conclude that each cycle in $G$ is of length at most $k-1$. Then by Lemma 6 , if $G$ has a circuit with at least $k$ arcs, then it has a circuit with at least $k$ and at most $2 k-2$ arcs. We find such a circuit (if it exist) by solving AT LEAST $k$ AND AT MOST $k$-CIRCUIT for $k^{\prime}=2 k-2$ by making use of Lemma 5 . Combining the running times, we have that LONG CIRCUIT can be solved in $2^{O(k \log k)} \cdot n m$ expected time and in $2^{O(k \log k)} \cdot n m \log n$ worst-case time.

For the undirected case, we assume that the input graph $G$ is connected, as otherwise we can solve the problem for each component. We choose a vertex $v$ arbitrarily and perform the depth-first search from $v$. In this way we find the fundamental cycles for the dfs-tree rooted in $v$, and check whether there is a fundamental cycle of length at least $k$. If we have such a cycle, then it is a circuit with at least $k$ edges, and we have a YES-answer. Otherwise, by Theorem 6 , either $G$ has no cycles of length at least $k$ or $G$ has a cycle with at least $k$ and at most $2 k-4$ edges. If $G$ has no cycles with at least $k$ edges, then by Lemma 6 , if $G$ has a circuit with at least $k$ edges, it contains a circuit with at least $k$ and at most $2 k-2$ edges. We conclude that if the constructed fundamental cycles have lengths at most $k-1$, then $G$ either has a circuit with at least $k$ and at most $2 k-2$ edges or has no circuit with at least $k$ edges. We check whether $G$ has a circuit with at least $k$ and at most $2 k-2$ edges by solving AT LEAST $k$ AND AT MOsT $k^{\prime}$-CIRCUIT for $k^{\prime}=2 k-2$ using Lemma 5. Since the depth-first search runs in linear time, we have that on undirected graphs LONG CiRCUIT can be solved in $2^{O(k)} \cdot n m$ expected time and in $2^{O(k)} \cdot n m \log n$ worst-case time.

\section{References}

[1] N. Alon, R. Yuster, And U. Zwick, Color-coding, J. ACM, 42 (1995), pp. 844-856.

[2] P. Berman, M. Karpinski, And A. D. Scott, Approximation hardness of short symmetric instances of MAX-3SAT, Electronic Colloquium on Computational Complexity (ECCC), (2003).

[3] H. L. Bodlaender, A linear-time algorithm for finding treedecompositions of small treewidth, SIAM J. Comput., 25 (1996), pp. $1305-1317$.

[4] H. L. Bodlaender, R. G. Downey, M. R. Fellows, and D. HerMELIN, On problems without polynomial kernels, J. Comput. Syst. Sci., 75 (2009), pp. 423-434. 
[5] L. Cai And B. Yang, Parameterized complexity of even/odd subgraph problems, J. Discrete Algorithms, 9 (2011), pp. 231-240.

[6] B. Courcelle, The monadic second-order logic of graphs III: treedecompositions, minor and complexity issues, ITA, 26 (1992), pp. 257286.

[7] M. Cygan, D. Marx, M. Pilipczuk, M. Pilipczuk, And I. Schlotter, Parameterized complexity of Eulerian deletion problems, in WG 2011, vol. 6986 of Lecture Notes Comp. Sci., Springer, 2011, pp. 131-142.

[8] F. Dorn, H. Moser, R. Niedermeier, And M. Weller, Efficient algorithms for eulerian extension and rural postman, SIAM J. Discrete Math.,, 27 (2013), pp. 75-94.

[9] R. G. Downey and M. R. Fellows, Parameterized complexity, Monographs in Computer Science, Springer-Verlag, New York, 1999.

[10] P. Erdös And G. Szekeres, A combinatorial problem in geometry, Compositio Math., 2 (1935), pp. 463-470.

[11] M. R. Fellows, D. Hermelin, F. A. Rosamond, And S. VialetTe, On the parameterized complexity of multiple-interval graph problems, Theor. Comput. Sci., 410 (2009), pp. 53-61.

[12] H. Fleischner, Eulerian Graphs and Related Topics, Part 1, Volume 1, Annals of Discrete Mathematics 45, Amsterdam, 1990.

[13] J. Flum and M. Grohe, Parameterized complexity theory, Texts in Theoretical Computer Science. An EATCS Series, Springer-Verlag, Berlin, 2006.

[14] F. V. Fomin and P. A. Golovach, Parameterized complexity of connected even/odd subgraph problems, in STACS 2012, vol. 14 of LIPIcs, Schloss Dagstuhl - Leibniz-Zentrum fuer Informatik, 2012, pp. 432-440.

[15] H. N. Gabow And S. NiE, Finding a long directed cycle, ACM Transactions on Algorithms, 4 (2008).

[16] M. R. Garey, D. S. Johnson, and R. E. Tarjan, The planar hamiltonian circuit problem is np-complete, SIAM J. Comput., 5 (1976), pp. $704-714$.

[17] P. A. Golovach And D. M. Thilikos, Paths of bounded length and their cuts: Parameterized complexity and algorithms, Discrete Optimization, 8 (2011), pp. 72-86. 
[18] A. Kosowski, B. Li, N. Nisse, And K. Suchan, k-chordal graphs: From cops and robber to compact routing via treewidth, in ICALP 2012, vol. 7392 of Lecture Notes in Computer Science, Springer, 2012, pp. $610-622$.

[19] R. Niedermeier, Invitation to fixed-parameter algorithms, vol. 31 of Oxford Lecture Series in Mathematics and its Applications, Oxford University Press, Oxford, 2006. 